\title{
Intra-aortic balloon counterpulsation in acute myocardial infarction: old and emerging indications
}

\author{
L. X. van Nunen • M. van 't Veer • S. Schampaert • \\ B. J. E. M. Steerneman • M. C. M. Rutten • \\ F. N. van de Vosse • N. H. J. Pijls
}

Published online: 30 October 2013

(C) The Author(s) 2013. This article is published with open access at Springerlink.com

\begin{abstract}
Background Recent evidence questions the role of intra-aortic balloon counterpulsation (IABP) in the treatment of acute myocardial infarction (AMI) complicated by cardiogenic shock (CS). An area of increasing interest is the use of IABP for persistent ischaemia (PI). We analysed the use of IABP in patients with AMI complicated by CS or PI.

Methods From 2008 to 2010, a total of 4076 patients were admitted to our hospital for primary percutaneous coronary intervention (PCI) for AMI. Out of those, 239 patients received an IABP either because of CS or because of PI. Characteristics and outcome of those patients are investigated.

Results The mean age of the study population was $64 \pm$ 11 years; $75 \%$ were male patients. Of the patients, $63 \%$ had CS and $37 \%$ had PI. Patients with CS had a 30-day mortality rate of $36 \%$; 1-year mortality was $41 \%$. Patients with PI had a 30-day mortality rate of $7 \%$; 1-year mortality was $11 \%$.

Conclusions Mortality in patients admitted for primary PCI because of AMI complicated by CS is high despite IABP use. Outcome in patients treated with IABP for PI is favourable and mandates further prospective studies.
\end{abstract}

L. X. van Nunen · M. van 't Veer · B. J. E. M. Steerneman •

N. H. J. Pijls

Department of Cardiology, Catharina Hospital Eindhoven,

Michelangelolaan 2, 5623 EJ Eindhoven, the Netherlands

L. X. van Nunen · M. van 't Veer · B. J. E. M. Steerneman •

N. H. J. Pijls $(\varangle)$

PO Box 1350, 5602 ZA Eindhoven, the Netherlands

e-mail: nico.pijls@inter.nl.net

S. Schampaert • M. C. M. Rutten · F. N. van de Vosse Department of Biomedical Engineering, Eindhoven University of Technology, PO Box 513, 5600 MB Eindhoven, the Netherlands
Keywords Intra-aortic balloon counterpulsation . Cardiogenic shock $\cdot$ Persistent ischaemia $\cdot$ Acute myocardial infarction

\section{Introduction}

Acute myocardial infarction (AMI) is complicated by cardiogenic shock (CS) in approximately $5-10 \%$ of patients $[1,2]$. Intra-aortic balloon counterpulsation (IABP) has been used as a haemodynamic support system for patients with AMI complicated by CS since 1968 [3]. Treatment with IABP is supposed to increase cardiac output by more effective emptying of the left ventricle, decreasing workload and thereby oxygen demand of the heart, and to reduce myocardial ischaemia by augmentation of coronary perfusion $[4,5]$.

Evidence for IABP use in patients with AMI complicated by CS is largely based upon pathophysiological considerations and non-randomised, small trials of patients treated with thrombolytic therapy [6]. However, the most recent large randomised trial on the use of IABP in patients with AMI complicated by CS showed no benefit on 30-day mortality [7]. As a result, the efficacy of IABP to treat patients with CS has been questioned.

In contrast, an area of increasing interest is the use of IABP in patients with persistent ischaemia (PI) after primary PCI for AMI (no-reflow phenomenon). Because PI is thought to be reversible over time (within hours to days), decreasing the workload of the myocardium and increasing coronary blood flow by IABP is suggested to limit the final size of the infarcted territory [8].

In this paper, we report our complete experience with the use of IABP in acute myocardial infarction, either as treatment for cardiogenic shock or to treat persistent ischaemia, over a period of 3 years. 


\section{Methods}

Patient population

All patients admitted to the Catharina Hospital Eindhoven for primary PCI in AMI (with ST elevation) during a 3-year period (1 January 2008 to 31 December 2010) were studied (Fig. 1). Of all patients, we analysed those who survived the interventional procedure and were treated by IABP. Baseline characteristics including cardiovascular risk factors, patient history, electrocardiograms, coronary anatomy, indication for IABP insertion, haemodynamic parameters at time of IABP insertion and possible complications were acquired from patient medical records and local databases.

Patients were retrospectively divided into two subgroups, based on the indication for treatment with IABP, i.e. CS or PI (without CS). CS was defined as a persistent state of hypotension (systolic blood pressure $<90 \mathrm{mmHg}$ or mean arterial pressure $30 \mathrm{mmHg}$ lower than baseline) and clinical signs of CS (abnormal mental status, cold clammy skin, oliguria). Patients in whom IABP was inserted because of mechanical complications of AMI (ventricular septal rupture, papillary muscle rupture) were assigned to the CS group by definition.

PI was defined as persistent or recurrent chest pain and ST elevation despite successful epicardial reperfusion therapy (no-reflow phenomenon), failed primary PCI with persistent chest pain and ST elevation, or bridge to CABG. Recurrent ST elevation was defined as return of ST elevation before leaving the catheterisation laboratory after initial regression due to successful reperfusion therapy.

Patients presenting with both CS and PI were assigned to the CS group by definition.

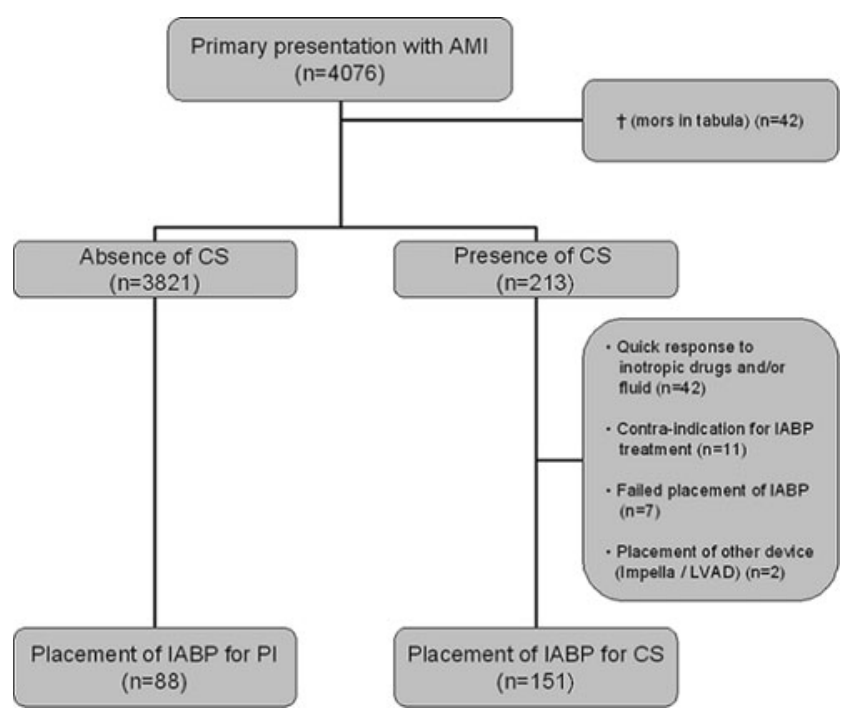

Fig. 1 Flowchart. Patients admitted for acute myocardial infarction to the Catharina Hospital Eindhoven, period 2008-2010
The Datascope 300 CS console, combined with the Maquet Sensation 7 Fr. $40 \mathrm{cc}$ balloon, was used in all patients (Maquet Inc, Wayne, NJ).

IABP-related complications were defined as peripheral ischaemia requiring IABP removal (absence of peripheral pulsation combined with white colouration of the leg on the side of IABP insertion), bleeding requiring transfusion or need for vascular surgery, and infection because of IABP insertion (fever and signs of inflammation, combined with increased Creactive protein levels $(>6 \mathrm{mg} / \mathrm{l}))$.

Follow-up and data collection

Follow-up was obtained at the time of IABP removal, hospital discharge, 30-day and 1-year follow-up. Outcome measures were all-cause mortality within 30 days and all-cause mortality at 1 year. Vital status was obtained by contacting the patient's treating cardiologist or general physician.

Statistical analysis

Discrete variables are presented as percentages, while continuous variables are presented as mean \pm standard deviations. Discrete variables were compared using the $X^{2}$ or Fisher's exact test as appropriate. Continuous variables were compared using the Student's $t$ test or Mann-Whitney$U$ test as appropriate.

Since hazard ratios of the covariates are not constant over time, we used multivariate regression analysis to identify predictors of 30-day and 1-year mortality.

The patients lost to follow-up were censored at the date of last contact (in all cases the date of hospital discharge).

A limited number of variables were preselected for multivariate regression analysis according to the size of the study population. The preselected variables were age, CS, cardiopulmonary resuscitation, use of antiarrhythmic drugs, use of inotropic agents and coronary artery bypass grafting (CABG).

The acquired data were analysed using IBM Statistical Package for Social Sciences (SPSS) for Windows Version 19.0.0.1 (IBM Corporation, Armonk, NY). All statistical tests were two-tailed and $p$-value $<0.05$ was considered statistically significant.

\section{Results}

Baseline characteristics and in-hospital outcome

In total, 4076 patients were admitted to the hospital for AMI. Of those patients, $42(1 \%)$ were moribund and died in the catheterisation laboratory and were excluded from this analysis. Overall in-hospital mortality was $2.6 \%$. In 239 
Table 1 Baseline characteristics of patients receiving intra-aortic balloon counterpulsation

\begin{tabular}{|c|c|c|c|c|}
\hline & \multirow[t]{2}{*}{ Total $(n=239)$} & \multicolumn{2}{|l|}{ Indication for IABP insertion } & \multirow[t]{2}{*}{$p$-value } \\
\hline & & Cardiogenic shock $(n=151)$ & Persistent ischaemia $(n=88)$ & \\
\hline Age (years) & $64 \pm 11$ & $64 \pm 11$ & $65 \pm 11$ & 0.87 \\
\hline Male sex $(\%(n))$ & $75(180)$ & $74(111)$ & $78(69)$ & 0.40 \\
\hline \multicolumn{5}{|l|}{ Risk factors } \\
\hline Hypertension $(\%(n))$ & $40(96)$ & $38(59)$ & $42(37)$ & 0.65 \\
\hline Diabetes mellitus $(\%(n))$ & $20(47)$ & $20(30)$ & $19(17)$ & 0.92 \\
\hline Hypercholesterolaemia $(\%(n))$ & $24(58)$ & $20(30)$ & $32(28)$ & 0.04 \\
\hline Smoking $(\%(n))$ & $32(77)$ & $31(47)$ & $34(30)$ & 0.58 \\
\hline Renal insufficiency $(\%(n))$ & $5(12)$ & $4(6)$ & $7(6)$ & 0.37 \\
\hline Peripheral arterial disease $(\%(n))$ & $8(20)$ & $7(10)$ & $11(10)$ & 0.20 \\
\hline \multicolumn{5}{|l|}{ History of } \\
\hline Coronary disease $(\%(n))$ & $32(77)$ & $29(43)$ & $39(34)$ & 0.11 \\
\hline CVA/TIA $(\%(n))$ & $9(22)$ & $9(14)$ & $9(8)$ & 0.96 \\
\hline Atrial fibrillation $(\%(n))$ & $3(8)$ & $4(6)$ & $2(2)$ & 0.71 \\
\hline Systolic blood pressure (mmHg) & $96 \pm 30$ & $80 \pm 27$ & $121 \pm 20$ & $<0.01$ \\
\hline Diastolic blood pressure (mmHg) & $55 \pm 18$ & $47 \pm 18$ & $68 \pm 14$ & $<0.01$ \\
\hline Heart rate (beats per minute) & $92 \pm 22$ & $95 \pm 25$ & $84 \pm 18$ & $<0.01$ \\
\hline Out of hospital cardiac arrest $(\%(n))$ & $25(60)$ & $32(48)$ & $14(12)$ & $<0.01$ \\
\hline Mechanical ventilation $(\%(n))$ & $23(55)$ & $29(44)$ & $13(11)$ & $<0.01$ \\
\hline \multicolumn{5}{|l|}{ Location of STEMI } \\
\hline Anterior/septal $(\%(n))$ & $43(103)$ & $40(61)$ & $48(42)$ & 0.27 \\
\hline Inferior/posterior $(\%(n))$ & $34(81)$ & $37(56)$ & $28(25)$ & 0.17 \\
\hline Undetermined/pan-ischaemia $(\%(n))$ & $22(53)$ & $22(33)$ & $22(20)$ & 0.88 \\
\hline Three vessel/left main stem coronary disease $(\%(n))$ & $62(147)$ & $61(92)$ & $63(55)$ & 0.86 \\
\hline Mechanical complication $(\%(n))$ & $8(20)$ & $13(20)$ & $0(0)$ & $<0.01$ \\
\hline \multicolumn{5}{|l|}{ Reperfusion therapy } \\
\hline Primary PCI $(\%(n))$ & 49 (118) & $59(89)$ & $33(29)$ & $<0.01$ \\
\hline CABG $(\%(n))$ & $38(91)$ & $33(50)$ & $47(41)$ & 0.04 \\
\hline No reperfusion or thrombolytic therapy $(\%(n))$ & $13(30)$ & $8(12)$ & $21(18)$ & $<0.01$ \\
\hline \multicolumn{5}{|l|}{ Indication for IABP-implantation } \\
\hline Reduction of ischaemia $(\%(n))$ & $37(88)$ & $0(0)$ & $100(88)$ & \\
\hline Cardiogenic shock $(\%(n))$ & $63(151)$ & $100(151)$ & $0(0)$ & \\
\hline \multicolumn{5}{|l|}{ Complications during hospitalisation } \\
\hline Use of inotropic agents $(\%(n))$ & $78(186)$ & $93(140)$ & $52(46)$ & $<0.01$ \\
\hline Use of antiarrhythmic agents $(\%(n))$ & $38(90)$ & $45(68)$ & $25(22)$ & $<0.01$ \\
\hline Renal failure (renal replacement therapy) $(\%(n))$ & $3(6)$ & $3(5)$ & $1(1)$ & 0.42 \\
\hline \multicolumn{5}{|l|}{ IABP running time } \\
\hline$\leq 1$ day $(\%(n))$ & $38(91)$ & $31(46)$ & $51(45)$ & $<0.01$ \\
\hline$\geq 1$ days $(\%(n))$ & $62(148)$ & $70(105)$ & $49(43)$ & $<0.01$ \\
\hline
\end{tabular}

Data are presented as mean $\pm \mathrm{SD}$ or percentages

CABG indicates coronary artery bypass grafting; $C V A$ cerebrovascular event, $I A B P$ intra-aortic balloon pump, $P C I$ percutaneous coronary intervention, STEMI ST-segment elevation myocardial infarction TIA transient ischemic attack

patients $(5.9 \%)$, an IABP was inserted at the catheterisation laboratory during or immediately following the revascularisation procedure (Fig. 1).

Reperfusion was achieved in $87 \%$ of the patients receiving IABP (study population), either by primary PCI
(49 \%) or by emergency CABG if primary PCI failed or was considered inferior to emergency CABG (38\%). Table 1 shows the baseline characteristics of these patients and the subgroups based upon indication for IABP insertion (CS or PI). 
Overall, patients had multiple risk factors for coronary artery disease. At the time of presentation, $25 \%$ of the patients were resuscitated and $23 \%$ were mechanically ventilated. In $63 \%$ of the patients, the indication for IABP placement was CS, in $37 \%$ PI. The baseline systolic and diastolic blood pressures were significantly higher in the patients treated for PI, while the need for inotropic agents (dobutamine and norepinephrine) was significantly lower. The IABP was used during $\leq 1$ day in $38 \%$ of the patients, $62 \%$ of the patients were supported by $\mathrm{IABP} \geq 1$ days.

There was a relatively low incidence of IABP-related complications of $4 \%$, due to major bleeding ( $2 \%$ ) and ischaemia ( $2 \%)$. Balloon rupture occurred in 1 patient.

Thirty-day and one-year follow-up

Follow-up at 30-days and 1-year was obtained in $98 \%$ of the patients. Four patients ( $2 \%$ ) were lost during follow-up due to transportation to their native country.

Cumulative 30-day mortality and 1-year mortality for patients treated with IABP was $26 \%$ and $31 \%$, respectively. Figure 2 shows the Kaplan-Meier survival curves of the two groups classified by indication of IABP use. The patients with CS had a 30-day mortality of $36 \%$ and a 1 -year mortality of $41 \%$. The patients treated with IABP because of PI had a 30day mortality of $7 \%$; 1 -year mortality was $11 \%$.

The results from the multivariate regression analyses are shown in Table 2. The presence of CS (OR, 4.7; $95 \%$ CI, 1.8
12.4) was independently associated with higher 30-day mortality.

For long-term survival, we performed a subanalysis of all patients successfully weaned from IABP after haemodynamic stabilisation (Table 2). Older age (OR, 1.04; $95 \%$ CI, 1.0 1.08) was independently associated with higher 1 -year mortality.

In both 30-day mortality (OR, $0.3 ; 95 \% \mathrm{CI}, 0.1-0.6)$ and 1year mortality (OR, $0.3 ; 95 \% \mathrm{CI}, 0.1-0.8)$, CABG was an independent predictor for better survival.

\section{Discussion}

This study describes IABP use in a population of consecutive patients over a 3-year period presenting with AMI, in whom either cardiogenic shock or persistent ischaemia was present after (successful or failed) primary PCI. It does not compare two groups of patients, but solely reflects on two different indications of IABP use. On the one hand, it confirms the poor outcome of CS, but at the same time, it opens the window for a potentially better treatment of another group of patients with a poor prognosis, i.e. patients with persistent ischaemia.

In patients with CS, 30-day survival was $64 \%$, in accordance with the existing literature [7,9].

In contrast, in patients with PI 30-day survival and 1-year survival were high ( $93 \%$ and $89 \%$, respectively). Although there are no randomised data in the literature to compare
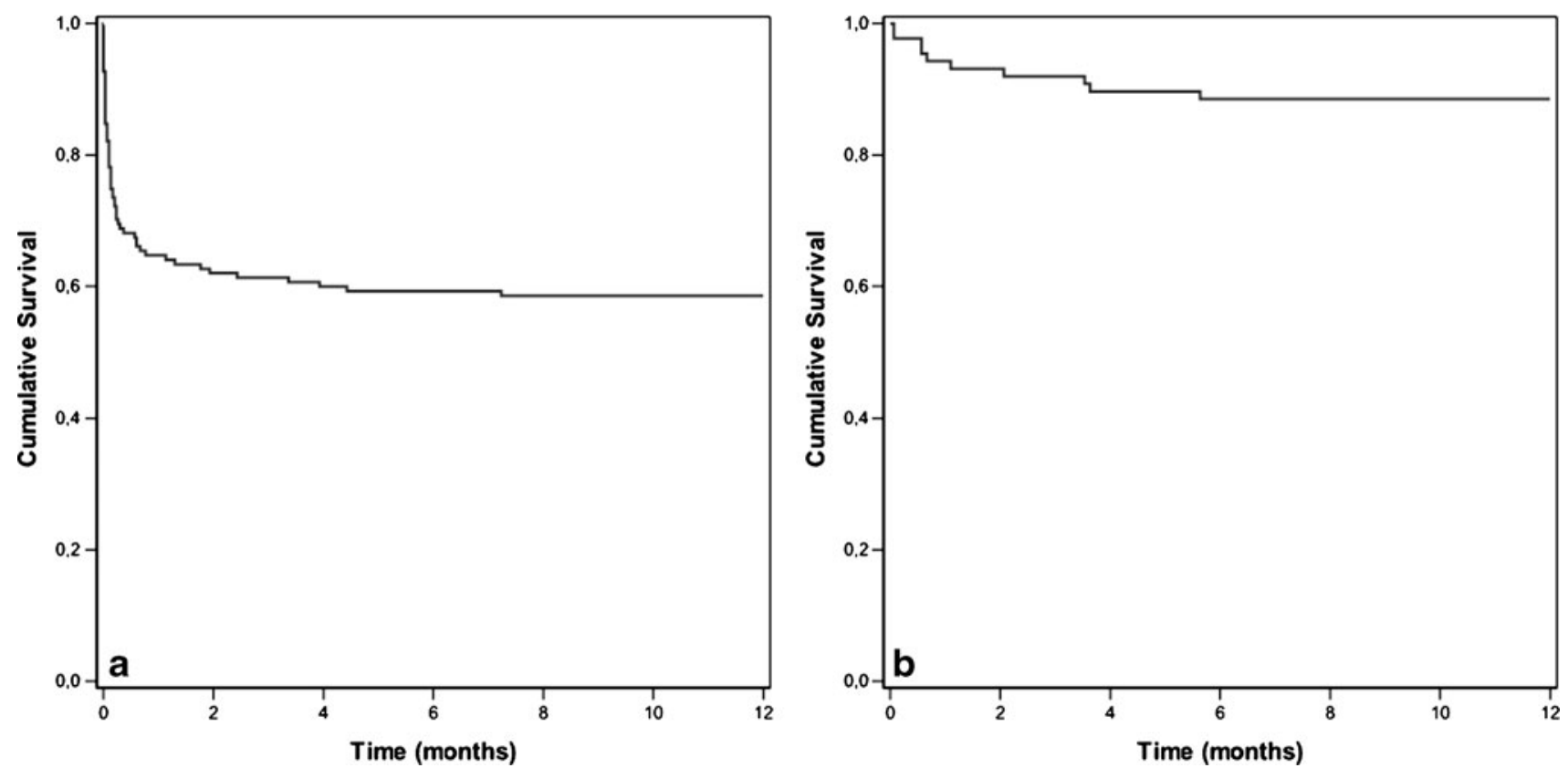

Fig. 2 One-year survival of patients treated with intra-aortic balloon counterpulsation. Panel A shows the Kaplan Meier curve of the patients treated with IABP for CS. Panel B shows the Kaplan Meier curve of the patients treated with IABP for PI 
Table 2 Multivariate regression analyses of 30-day survival in all patients treated with IABP and 1year survival in patients who survived until weaning of IABP

$\mathrm{CABG}$ indicates coronary artery bypass grafting; $C I$ confidence intervals, $C P R$ cardiopulmonary resuscitation, $I A B P$ intra-aortic balloon pump, $O R$ odds ratio

\begin{tabular}{|c|c|c|c|c|c|c|}
\hline & \multicolumn{3}{|c|}{ 30-day follow up } & \multicolumn{3}{|c|}{ 1-year follow up } \\
\hline & OR & $95 \% \mathrm{CI}$ & $p$-value & OR & $95 \%$ CI & $p$-value \\
\hline Age & 1.03 & $1.00-1.06$ & 0.06 & 1.04 & $1.0-1.08$ & 0.03 \\
\hline Cardiogenic shock & 4.7 & $1.8-12.4$ & 0.002 & 1.8 & $0.7-4.6$ & 0.19 \\
\hline CPR & 1.8 & $0.8-3.7$ & 0.13 & 2.2 & $1.0-5.3$ & 0.06 \\
\hline Anti-arrhythmic drugs & 1.1 & $0.5-2.2$ & 0.80 & 1.2 & $0.5-2.7$ & 0.65 \\
\hline Inotropic agents & 2.9 & $0.9-9.7$ & 0.08 & 2.6 & $0.7-8.9$ & 0.14 \\
\hline CABG & 0.3 & $0.1-0.6$ & 0.002 & 0.3 & $0.1-0.8$ & 0.01 \\
\hline
\end{tabular}

survival of patients treated with IABP for PI, survival of patients with PI treated medically is much worse $[10,11]$.

Reperfusion by CABG was independently associated with better 30-day survival, most likely because of selection bias through acceptance for emergency $\mathrm{CABG}$.

In this study, there were few complications of IABP use (4\%), comparable with the percentage of complications in the IABP-SHOCK II trial.

\section{IABP as treatment of cardiogenic shock}

Patients treated with IABP in our study who met the criteria of CS had a 30-day survival of $64 \%$, which is in line with the recent IABP-SHOCK II trial, showing a 30-day survival of $61 \%$. These survival rates are high when compared with other, older studies $[12,13]$, most likely because of the high percentage of early revascularisation $(90 \%)[14,15]$. The IABP-SHOCK II trial was the first randomised controlled trial on the use of IABP in patients presenting with AMI complicated by CS where reperfusion was achieved mostly by PCI and showed no reduction in short-term mortality [7]. This landmark study has some limitations which were discussed recently [16]. The study was underpowered due to the 30-day mortality which was significantly lower than expected. Also, there was a relatively high rate of crossover in the control group (10\%) and there was a trend towards an increase in the use of left ventricular assist devices in the control group. These limitations are inherent in such a study in 'back-against-the-wall' patients, but could mask the potential benefit of IABP use.

Nonetheless, these results question if IABP deserves its place as routine treatment of patients with AMI complicated by CS, but do not exclude a beneficial effect in some patients. The challenge is to find a way to distinguish between those patients and not abandon such a potentially life-saving treatment regardlessly. At least, it can be expected that a beneficial effect of augmented diastolic pressure only plays a role in case of PI with exhausted autoregulation. In case of CS without PI, improved coronary blood flow is unlikely.
IABP as treatment of persistent ischaemia

Use of IABP after successful epicardial reperfusion complicated by PI (no-reflow phenomenon), or after failed PCI, is an area which has not been investigated extensively. This no-reflow phenomenon is attributable to a variety of factors, including micro-embolisation, spasm, intramyocardial oedema and other entities incompletely understood [17], but leading to enlargement of the area of myocardial necrosis.

In a number of these patients, dramatic relief of ischaemia occurs after insertion of IABP, most likely due to afterload reduction and augmentation of myocardial blood flow. Since coronary autoregulation is completely exhausted in these patients, myocardial blood flow is directly dependent on perfusion pressure. Because of that exhausted flow reserve, a positive effect of augmented perfusion pressure is well conceivable [18].

The prognosis in patients with PI treated medically is poor. Rezkalla et al. described a 30-day mortality of $12 \%$ in 64 patients who suffered from severely impaired myocardial blush grade after PCI for AMI [11]. Mehta et al. confirmed this poor prognosis with a 1 -year mortality of $23 \%$ in patients with STEMI undergoing primary PCI with final TIMI $\leq 2$ flow [10]. Because of this poor prognosis, the value of attempts to relieve PI is undisputable.

In our study, patients treated with IABP for PI had a favourable 30-day survival of $93 \%$ and a 1-year survival of $89 \%$. Since this study was performed retrospectively, there is no control group. Outcome data can therefore only be compared with the historical data, but are favourable and mandate further randomised studies in such patients with STEMI undergoing primary PCI complicated by PI.

Favourable long-term survival rates have been described after IABP support in myocardial infarctions in nonrandomised studies [19]. A randomised trial (CRISP-AMI) on adjunctive IABP therapy to limit infarct size in patients with AMI in the absence of CS, failed to show benefit of IABP treatment [20]. All-cause mortality at 6 months was less in the IABP group ( $1.9 \%$ versus $5.2 \% ; P=0.12)$, but was not statistically significant due to the low rate of events (underpowered study). 
We believe IABP should not be used regularly in every patient presenting with AMI, but there might be a subset of patients - those with PI or no-reflow - who might benefit from IABP support. In the presence of PI, this potentially detrimental phenomenon might be bridged by using IABP to reduce workload and oxygen demand of the myocardium and increase myocardial perfusion. In this context, it has been speculated in the literature whether timing of initiation of IABP support is important, i.e. just before or after opening of the occluded artery. A recent study with respect to timing did not show any significant differences in odds of mortality [21].

\section{Limitations}

This study is a retrospective analysis of all AMI patients but with two fundamentally different characteristics (CS and PI). There are no matched-control groups and the results of IABP treatment in both groups had to be compared with historical data. Furthermore, in the setting of no-reflow, additional medical therapy is sometimes suggested, such as abciximab or verapamil. In our study, none of these drugs were systematically investigated. Verapamil was used in 4 patients, while GP IIb/IIIa inhibitors were used only in 13 patients because of angiographic signs of distal embolisation. Nevertheless, this analysis of IABP use includes all consecutive patients with AMI during 3 years within one large heart centre, is not limited to the traditional CS patients but also studied systematically patients with PI and is hypothesisgenerating for the future.

\section{Conclusions}

In this large retrospective study in 4076 consecutive patients presenting with acute myocardial infarction during 3 years, IABP was used in $6 \%(n=239)$ of all cases because of two different indications: cardiogenic shock $(3.8 \%)$ or persistent ischaemia $(2.2 \%)$. Mortality in patients with cardiogenic shock was high - but comparable with recent literature. Outcome in patients with persistent ischaemia was favourable when compared with historical data, and mandates further prospective studies.

\section{Acknowledgments Dr. Pijls receives research grants from Maquet.}

Funding This work was supported by the Dutch Technology Foundation STW (Stichting voor de Technische Wetenschappen) under project number 11052

Open Access This article is distributed under the terms of the Creative Commons Attribution License which permits any use, distribution, and reproduction in any medium, provided the original author(s) and the source are credited.

\section{References}

1. Babaev A, Frederick PD, Pasta DJ, et al. Trends in management and outcomes of patients with acute myocardial infarction complicated by cardiogenic shock. JAMA. 2005;294:448-54.

2. Goldberg RJ, Spencer FA, Gore JM, et al. Thirty-year trends (1975 to 2005 ) in the magnitude of, management of, and hospital death rates associated with cardiogenic shock in patients with acute myocardial infarction: a population-based perspective. Circulation. 2009;119: $1211-9$

3. Kantrowitz A, Tjonneland S, Freed PS, et al. Initial clinical experience with intraaortic balloon pumping in cardiogenic shock. JAMA. 1968;203:113-8.

4. Kern MJ, Aguirre FV, Tatineni S, et al. Enhanced coronary blood flow velocity during intraaortic balloon counterpulsation in critically ill patients. J Am Coll Cardiol. 1993;21:359-68.

5. Prondzinsky R, Unverzagt S, Russ $M$, et al. Hemodynamic effects of intra-aortic balloon counterpulsation in patients with acute myocardial infarction complicated by cardiogenic shock: the prospective, randomized IABP shock trial. Shock. 2012;37: 378-84.

6. Sjauw KD, Engstrom AE, Vis MM, et al. A systematic review and meta-analysis of intra-aortic balloon pump therapy in ST-elevation myocardial infarction: should we change the guidelines? Eur Heart J. 2009;30:459-68.

7. Thiele H, Zeymer U, Neumann FJ, et al. Intraaortic balloon support for myocardial infarction with cardiogenic shock. N Engl J Med. 2012;367:1287-96.

8. Pierrakos CN, Bonios MJ, Drakos SG, et al. Mechanical assistance by intra-aortic balloon pump counterpulsation during reperfusion increases coronary blood flow and mitigates the no-reflow phenomenon: an experimental study. Artif Organs. 2011;35:867-74.

9. Cheng JM, Valk SD, den Uil CA, et al. Usefulness of intraaortic balloon pump counterpulsation in patients with cardiogenic shock from acute myocardial infarction. Am J Cardiol. 2009;104:327-32.

10. Mehta RH, Harjai KJ, Cox D, et al. Clinical and angiographic correlates and outcomes of suboptimal coronary flow in patients with acute myocardial infarction undergoing primary percutaneous coronary intervention. J Am Coll Cardiol. 2003;42:1739-46.

11. Rezkalla SH, Dharmashankar KC, Abdalrahman IB, et al. Noreflow phenomenon following percutaneous coronary intervention for acute myocardial infarction: incidence, outcome, and effect of pharmacologic therapy. J Intery Cardiol. 2010;23:429-36.

12. Anderson RD, Ohman EM, Holmes Jr DR, et al. Use of intraaortic balloon counterpulsation in patients presenting with cardiogenic shock: observations from the GUSTO-I study. Global utilization of streptokinase and TPA for occluded coronary arteries. J Am Coll Cardiol. 1997;30:708-15.

13. Barron HV, Every NR, Parsons LS, et al. The use of intra-aortic balloon counterpulsation in patients with cardiogenic shock complicating acute myocardial infarction: data from the National Registry of Myocardial Infarction 2. Am Heart J. 2001;141:933-9.

14. Steg PG, James SK, Atar D, et al. ESC guidelines for the management of acute myocardial infarction in patients presenting with ST-segment elevation: the task force on the management of ST-segment elevation acute myocardial infarction of the European Society of Cardiology (ESC). Eur Heart J. 2012;33:2569-619.

15. Antman EM, Anbe DT, Armstrong PW, et al. ACC/AHA guidelines for the management of patients with ST-elevation myocardial infarction; A report of the American College of Cardiology/ 
American Heart Association Task Force on Practice Guidelines (Committee to Revise the 1999 Guidelines for the Management of patients with acute myocardial infarction). J Am Coll Cardiol. 2004;44:E1-211.

16. Perera D, Lumley M, Pijls NH, et al. Intra-aortic balloon pump trials: questions, answers, and unresolved issues. Circ Cardiovasc Interv. 2013;6:317-21.

17. Rezkalla SH, Kloner RA. No-reflow phenomenon. Circulation. 2002;105:656-62.

18. Pijls NH, De Bruyne B. Coronary pressure measurement and fractional flow reserve. Heart. 1998;80:539-42.
19. Valk SD, Cheng JM, den Uil CA, et al. Encouraging survival rates in patients with acute myocardial infarction treated with an intra-aortic balloon pump. Neth Heart J. 2011;19:112-8.

20. Patel MR, Smalling RW, Thiele H, et al. Intra-aortic balloon counterpulsation and infarct size in patients with acute anterior myocardial infarction without shock: the CRISP AMI randomized trial. JAMA. 2011;306:1329-37.

21. Sjauw KD, Engstrom AE, Vis MM, et al. Efficacy and timing of intra-aortic counterpulsation in patients with ST-elevation myocardial infarction complicated by cardiogenic shock. Neth Heart J. 2012;20: 402-9. 